\title{
Components of strong magnetoresistance in Mn implanted Ge
}

\author{
A. Simons, ${ }^{1}$ A. Gerber, ${ }^{1, a)}$ I. Ya. Korenblit, ${ }^{1}$ A. Suslov, ${ }^{2}$ B. Raquet, ${ }^{3}$ M. Passacantando, ${ }^{4}$ \\ L. Ottaviano, ${ }^{4}$ G. Impellizzeri, ${ }^{5}$ and B. Aronzon ${ }^{6}$ \\ ${ }^{1}$ Raymond and Beverly Sackler Faculty of Exact Sciences, School of Physics and Astronomy, \\ Tel Aviv University, Ramat Aviv 69978 Tel Aviv, Israel \\ ${ }^{2}$ National High Magnetic Field Laboratory, 1800 East Paul Dirac Drive, Tallahassee, \\ Florida 32310-3706, USA \\ ${ }^{3}$ Laboratoire National des Champs Magnétiques Pulsés (LNCMP), 143 Av. de Rangueil, \\ 31432 Toulouse, France \\ ${ }^{4}$ Dipartimento di Fisica, Università degli Studi dell' Aquila, Via Vetoio, 67100 Coppito (AQ), Italy \\ ${ }^{5}$ CNR_INFM, CRS MATIS, Dipartimento di Fisica e Astronomia, Università di Catania, \\ Via Santa Sofia 64, 95123 Catania, Italy \\ ${ }^{6}$ Institute of Theoretical and Applied Electrodynamics, Russian Academy of Sciences, \\ Izhorskaya Str. 13, 125412 Moscow, Russia
}

(Received 20 November 2013; accepted 22 February 2014; published online 5 March 2014)

\begin{abstract}
Strong magnetoresistance reaching thousands of percent and non-monotonic field dependent Hall effect were measured in $\mathrm{Mn}$ implanted Ge samples in fields up to $60 \mathrm{~T}$ and analyzed in the framework of a two carriers model. The measured Hall effect and temperature dependent zero field resistance can be consistently described by parallel conductance along thick p-type Ge substrate with low concentration of highly mobile carriers and along thin Mn doped Ge layer with low mobility carriers. However, the same model is not sufficient to explain experimentally observed suppression of quadratic field dependence of magnetoresistance at low fields and absence of its saturation at high fields. Two additional mechanisms were identified: a strong quasi-linear magnetoresistance of Ge substrate that dominates the low field range and a nonsaturating " $3 / 2$ " power law magnetoresistance of the Mn doped Ge layer dominating the high field range. (C) 2014 AIP Publishing LLC. [http://dx.doi.org/10.1063/1.4867623]
\end{abstract}

\section{INTRODUCTION}

Development of spintronics and prospects to reinforce the semiconducting electronics by the spin-dependent degree of freedom has triggered the recent interest in ferromagnetic semiconductors. Germanium doped with $\mathrm{Mn}$ is particularly interesting since ferromagnetism in this system is predicted to develop at high temperatures and the material is fully compatible with the existing silicon technology. GeMn films are usually fabricated by molecular beam epitaxial codeposition of Ge and Mn on Ge or GaAs substrates, or by Mn implantation of $\mathrm{Ge}$ wafers with or without post-fabrication annealing. Due to the very low solubility limit of Mn in germanium, ${ }^{1} \mathrm{Mn}$ segregation is unavoidable, and doped films are strongly inhomogeneous with $\mathrm{Ge}_{8} \mathrm{Mn}_{11},{ }^{2} \mathrm{Ge}_{3} \mathrm{Mn}_{5},{ }^{3-5}$ or $\mathrm{Ge}_{2} \mathrm{Mn}_{5}$ (Ref. 6) metallic precipitates coexisting with Mn-rich regions and $\mathrm{Mn}$ dilute matrix. Exact composition and topology of $\mathrm{Mn}$ rich precipitates depends on fabrication techniques.

Despite variations among samples grown by different technologies, a number of common magnetotransport effects were reported. The most striking of them are a huge positive magnetoresistance, reaching thousands of percent, and a non-monotonic, sign reversing field dependence of Hall effect. Several interpretations of the phenomena have been suggested. In Refs. 7 and 8, the positive MR was ascribed to geometrically enhanced orbital MR as a consequence of the

\footnotetext{
a) Author to whom correspondence should be addressed. Electronic mail: gerber@post.tau.ac.il.
}

conductivity contrast between the high-conductivity Mn-rich nanocolumns and the surrounding low-conductivity doped germanium matrix. Scattering off the spatial fluctuations of Mn atomic spin concentrations was suggested in Refs. 9 and 10. Localization of charge carriers due to magnetization induced transition from metallic to the insulating state in semiconductors with strongly inhomogeneous distribution of magnetic moments has been proposed in Refs. 11 and 12 . Less exotic mechanism was suggested by Zhou et al. ${ }^{13}$ that pointed out that much of the phenomena, in particular nonmonotonous Hall angle, can be explained by parallel conduction of two charge carriers generated by, e.g., two-level Mn acceptors in Ge. Supporting evidence for the two-carrier transport, although in a different geometrical implementation, was demonstrated by Sircar et al. ${ }^{14}$ that showed that parallel conduction along a doped layer and Ge substrate can generate very large magnetoresistance and non-monotonic Hall effect remarkably similar to the previous GeMn data, also when non-magnetic GeB films were grown on top of $\mathrm{Ge}$ substrates. It was thus concluded that spectacular properties of GeMn systems are not a consequence of alloying of magnetic Mn with Ge but result from parallel conduction along thin conducting doped epilayer and thick resistive $\mathrm{Ge}$ substrate.

In this work, we made a detailed analysis of several Mn doped Ge samples in an attempt to clarify whether the framework of two- or three-carriers transport in two parallel conducting layers is sufficient to reproduce complexity of the phenomena observed in GeMn systems. We shall show that while the temperature dependence of resistivity and Hall 
effect can be well described by the two layers model, the latter fails in explaining the observed magnetoresistance both in the low and high field limits. We identify two additional ingredients of magnetoresistance: a strong quasi-linear magnetoresistance of $\mathrm{Ge}$ substrate that dominates the low field range and a non-saturating power law magnetoresistance of the Mn doped Ge layer dominating the high field range. No adequate explanation of both phenomena has been found so far.

\section{EXPERIMENTAL}

Two Ge:Mn samples we will discuss here were fabricated by implanting $\mathrm{Mn}^{+}$ions into commercial single-crystalline $\mathrm{Ge}(100)$ wafers $0.35 \mathrm{~mm}$ thick with resistivity of $40-57 \Omega \mathrm{cm} . \mathrm{Mn}^{+}$ions were implanted with an energy of $100 \mathrm{keV}$ at fluences of $1 \cdot 10^{16}$ and $2 \cdot 10^{16}$ that produce samples with average volume concentrations of $\mathrm{Mn}$ of about 2 at. \% (denoted as $\mathrm{GeMn}_{2}$ ) and 4 at. \% (denoted as $\mathrm{GeMn}_{4} \mathrm{~A}$ ) in the projected depth range of about $120 \mathrm{~nm}$. During the implantation, the samples were held at $300^{\circ} \mathrm{C}$ to avoid amorphization. $\mathrm{GeMn}_{4} \mathrm{~A}$ sample has been annealed at $400 \mathrm{C}$ after the implantation. Structure of the samples is strongly nonuniform, depending on the concentration, and contains diluted $\mathrm{Mn}$, amorphous semiconducting Mn-rich nanoclusters, and ferromagnetic metallic $\mathrm{Mn}_{5} \mathrm{Ge}_{3}$ clusters. Detailed structural, chemical, and magnetic analyses of these samples can be found in Refs. 15 and 16. In addition, we measured a reference Ge substrate sample cut from a commercial wafer used for implantation. Ge substrate and $\mathrm{GeMn}_{2}$ samples had Hall bar geometry, while $\mathrm{GeMn}_{4} \mathrm{~A}$ sample was measured in the Van der Pauw configuration. Most of the measurements were done in fields up to $17 \mathrm{~T}$ at Tel Aviv. Sample $\mathrm{GeMn}_{2}$ was also measured in static fields up to $33 \mathrm{~T}$ at NHMFL at Tallahassee and up to $60 \mathrm{~T}$ in the pulsed magnetic field installation at Toulouse.

\section{RESULTS AND DISCUSSION}

Fig. 1 presents the temperature dependence of resistivity measured at zero magnetic field in three selected samples: $\mathrm{GeMn}_{2}, \mathrm{GeMn}_{4} \mathrm{~A}$, and Ge substrate. All samples behave similar above $20 \mathrm{~K}$ : resistivity decreases with decreasing temperature down to a minimum at about $25 \mathrm{~K}$ while the minimal resistances of all samples are very close to each other. Below the freezing temperature, resistivity of Ge substrate diverges to infinity while resistivity of Mn doped samples roughly stabilizes below $10 \mathrm{~K}$. The low temperature range is shown in the inset of Fig. 1. Resistance of Ge substrate at low temperatures can be well presented by an exponential divergence: $R=R_{0} \exp \left(E_{a} / T\right)$ with activation energy $E_{a} \approx 10.9 \mathrm{meV}$ which is in a good agreement with known values for shallow acceptors in Ge. ${ }^{17}$ One can therefore conclude that Ge substrate provides a major conducting channel in all three samples down to its freezing temperature. At $4 \mathrm{~K}$, Ge substrate is insulating and GeMn doped layer is the only conducting channel in the implanted samples.

Figs. 2 and 3 present two major characteristic properties of Mn doped Ge systems that triggered this study: a huge positive magnetoresistance reaching $12000 \%$ in $\mathrm{GeMn}_{4} \mathrm{~A}$

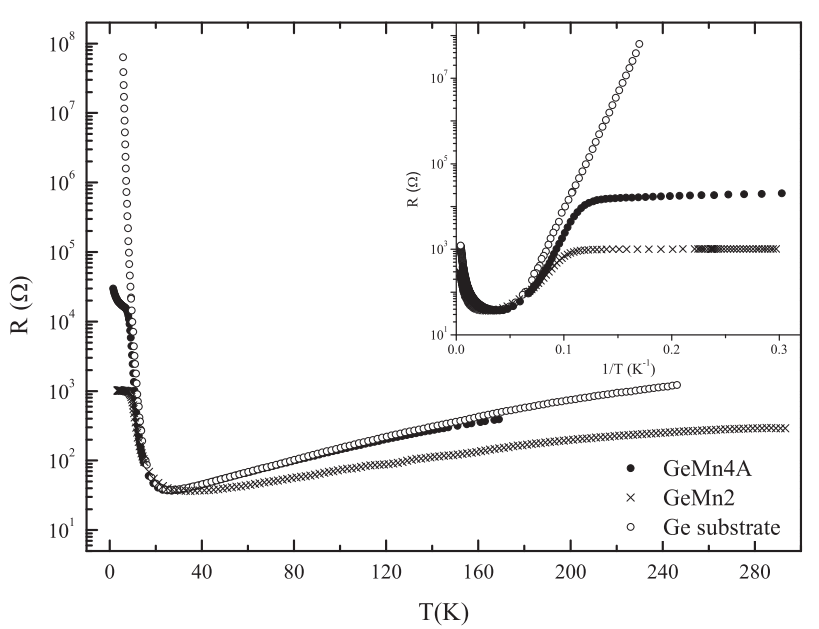

FIG. 1. Temperature dependence of resistivity measured at zero magnetic field in three samples: $\mathrm{GeMn}_{2}, \mathrm{GeMn}_{4} \mathrm{~A}$, and Ge substrate.

sample at 16 Tesla at $20 \mathrm{~K}$ (resistance changing from $41 \Omega$ at zero field to $5000 \Omega$ at $16 \mathrm{~T}$ ) and a non-monotonic field dependence of Hall resistance, sharply emphasized below $15 \mathrm{~K}$. In the following, we shall analyze these data in the framework of parallel conduction along Ge substrate and the doped MnGe layer but, differently from Refs. 13 and 14, the magnetoresistance and Hall effect will be treated separately.

\section{TWO CARRIERS/TWO LAYERS MODEL}

In its simple form, the two carriers model assumes that each of the carriers obeys the Bolzmann statistics with time interval between scattering events not depending on the carrier's energy. Field dependent longitudinal resistivity $\rho_{x x}(B)$ of the system can then be presented as

$$
\rho_{x x}(B)=\frac{\sigma_{1}+\sigma_{2}+\sigma_{1} \sigma_{2}\left(\sigma_{1} R_{1}^{2}+\sigma_{2} R_{2}^{2}\right) B^{2}}{\left(\sigma_{1}+\sigma_{2}\right)^{2}+\sigma_{1}^{2} \sigma_{2}^{2}\left(R_{1}+R_{2}\right)^{2} B^{2}}
$$

and the transverse (Hall) resistivity $\rho_{x y}$ is given by

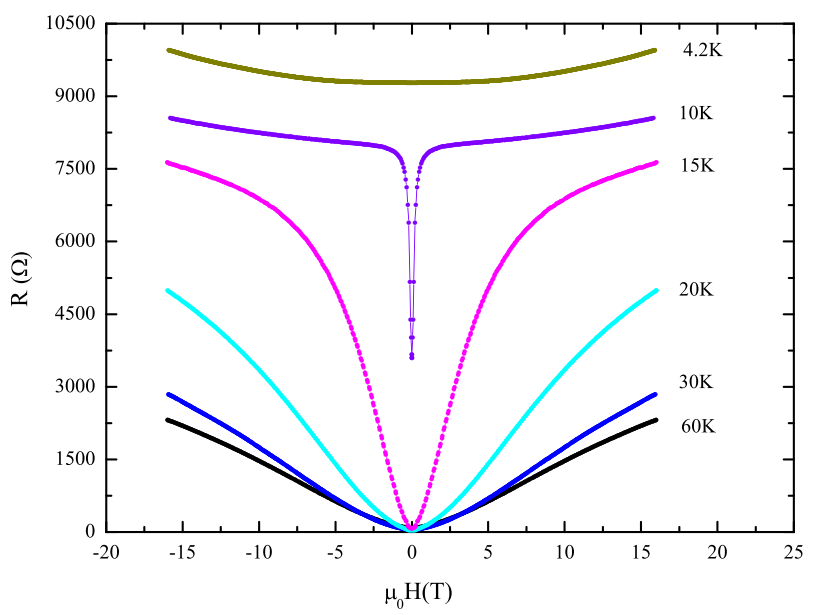

FIG. 2. Resistance of $\mathrm{GeMn}_{4} \mathrm{~A}$ sample as a function of magnetic field at several selected temperatures. Magnetic field is applied perpendicular to the sample plane. 


$$
\rho_{x y}(B)=\frac{R_{1} \sigma_{1}^{2}+R_{2} \sigma_{2}^{2}+R_{1} R_{2} \sigma_{1}^{2} \sigma_{2}^{2}\left(R_{1}+R_{2}\right) B^{2}}{\left(\sigma_{1}+\sigma_{2}\right)^{2}+\sigma_{1}^{2} \sigma_{2}^{2}\left(R_{1}+R_{2}\right)^{2} B^{2}} B
$$

where $\sigma_{1}$ and $\sigma_{2}$ are conductivities and $R_{1}$ and $R_{2}$ are Hall factors $\left(R=\frac{d \rho_{x y}}{d B}\right)$ of two carriers respectively. Mobility $\mu_{i}$ and density $n_{i}$ of each carrier $(\mathrm{i}=1,2)$ can be calculated from $\sigma_{i}$ and $R_{i}$ as: $n_{i}=\frac{1}{R_{i} e}$ and $\mu_{i}=\sigma_{i} \cdot R_{i}$. There are four unknown parameters in this presentation: $\sigma_{1}, \sigma_{2}, R_{1}$, and $R_{2}$. By changing variables, Eq. (1) can be presented in the form of the normalized magnetoresistivity as

$$
\frac{\rho_{x x}(B)-\rho_{0}}{\rho_{0}}=\frac{\rho_{\infty}-\rho_{0}}{\rho_{0}} \cdot \frac{\left(B / B_{s}\right)^{2}}{1+\left(B / B_{s}\right)^{2}},
$$

where resistivities $\rho_{1}\left(\rho_{1}=1 / \sigma_{1}\right)$ and $\rho_{2}\left(\rho_{2}=1 / \sigma_{2}\right)$ contributed by two carriers separately give rise to the zero field resistivity $\rho_{0}$ as

$$
\rho_{0}=\frac{1}{\sigma_{1}+\sigma_{2}}=\frac{\rho_{1} \rho_{2}}{\rho_{1}+\rho_{2}} .
$$

Resistivity of the system at infinitely high field $\rho_{\infty}$ is given by

$$
\rho_{\infty}=\frac{R_{1}^{2} \rho_{1}+R_{2}^{2} \rho_{2}}{\left(R_{1}+R_{2}\right)^{2}}
$$

and a characteristic scaling field $B_{s}$ is given by

$$
B_{s}=\frac{\rho_{1}+\rho_{2}}{R_{1}+R_{2}} .
$$

Respectively, the transverse (Hall) resistivity can be presented as

$$
\rho_{x y}=R_{0} \frac{1+\left(R_{\infty} / R_{0}\right)\left(B / B_{s}\right)^{2}}{1+\left(B / B_{s}\right)^{2}} B
$$

where $R_{0}$ and $R_{\infty}$ are the zero and infinite field Hall factors, given by

$$
R_{0}=\frac{R_{1} \sigma_{1}^{2}+R_{2} \sigma_{2}^{2}}{\left(\sigma_{1}+\sigma_{2}\right)^{2}}=\frac{R_{1} \rho_{2}^{2}+R_{2} \rho_{1}^{2}}{\left(\rho_{1}+\rho_{2}\right)^{2}}
$$

and

$$
R_{\infty}=\frac{R_{1} R_{2}}{R_{1}+R_{2}}
$$

$\rho_{0}$ and $R_{0}$ are experimentally measured quantities, therefore Eqs. (3) and (4) contain only three free parameters: $\rho_{\infty}, R_{\infty}$, and $B_{s}$ as compared with four free parameters $\sigma_{1}, \sigma_{2}, R_{1}$, and $R_{2}$ in Eqs. (1) and (2). We made fitting of the transverse resistivity $\rho_{x y}(B)$ data by Eq. (4) using the measured $R_{0}$ values and the fitting parameters $R_{\infty}$ and $B_{s}$. The magnetoresistance was fitted by Eq. (3) using the measured $\rho_{0}$ and fitting parameters $\rho_{\infty}$ and $B_{s}$.

Two features of the model will be important for the further discussion: magnetoresistance is expected to be parabolic at low fields

$$
\frac{\rho_{x x}(B)-\rho_{0}}{\rho_{0}}=\frac{\rho_{\infty}-\rho_{0}}{\rho_{0} B_{s}^{2}} B^{2} \quad\left(B \ll B_{s}\right)
$$

and to saturate to a constant value

$$
\frac{\rho_{x x}(B)-\rho_{0}}{\rho_{0}}=\frac{\rho_{\infty}-\rho_{0}}{\rho_{0}}
$$

at high fields when $B \gg B_{s}$.

\section{FITTING OF THE HALL EFFECT DATA}

Fig. 3(a) presents the Hall resistance measured in sample $\mathrm{GeMn}_{4} \mathrm{~A}$ at relatively low temperatures where the non-monotonic field dependence is well pronounced in the measured field range. We model the sample as composed of two separate conducting layers: Ge substrate $0.35 \mathrm{~mm}$ thick and $\mathrm{Mn}$ doped Ge layer $150 \mathrm{~nm}$ thick. The fitted curves reproduce the measured data well, in particular at higher temperatures where the signal varies smoothly. Parameters derived from the Hall data fitting at $30 \mathrm{~K}$ are: $B_{S}=11.1 T$ and $R_{\infty}=93 \Omega / T$ with the measured $R_{0}=345 \Omega / T$. At $10 \mathrm{~K}$, the respective values are: $B_{s}=0.2 T$ and $R_{\infty}=61$ $\Omega / T$ with the measured $R_{0}=18400 \Omega / T$. Fig. 3(b) presents
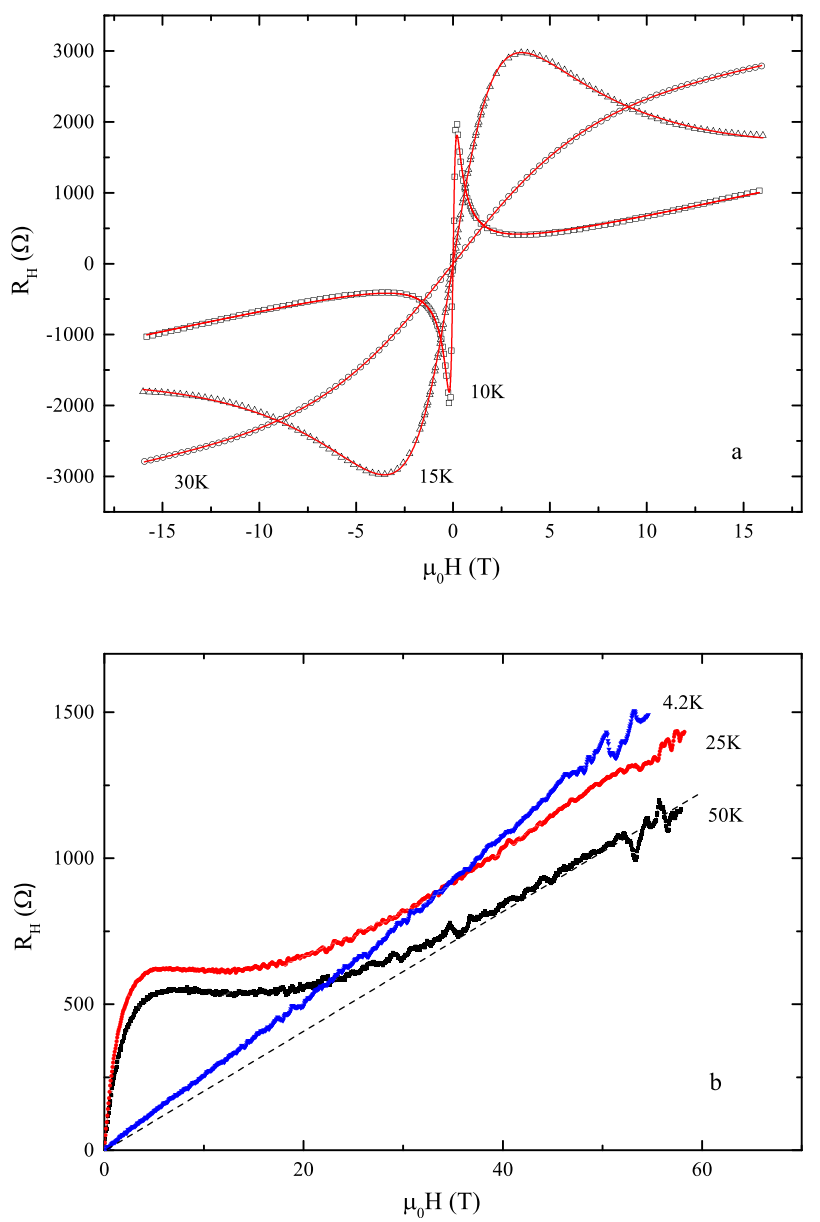

FIG. 3. (a) Hall resistance measured in $\mathrm{GeMn}_{4} \mathrm{~A}$ sample at three selected temperatures $(10 \mathrm{~K}, 15 \mathrm{~K}, 30 \mathrm{~K})$. The solid lines are fits calculated by Eq. (4). (b) The high field Hall resistances measured at several temperatures $(4.2 \mathrm{~K}, 25 \mathrm{~K}, 50 \mathrm{~K})$ in $\mathrm{GeMn}_{2}$ sample. The dotted line is guide for eye indicating that extrapolation of the linear high field slope crosses zero at zero field. 
the Hall resistance of $\mathrm{GeMn}_{2}$ sample measured at few temperatures in pulsed fields up to $60 \mathrm{~T}$. These high field measurements are less accurate than those taken at lower static fields; however, they allow us to confirm two important points:

(i) at temperatures up to $50 \mathrm{~K}$, the Hall slope becomes strictly linear at high fields in the range 30-60 T. Following the model, the Hall slope in the high field limit is $R_{\infty}=\frac{R_{1} R_{2}}{R_{1}+R_{2}}$. Thus, the slopes measured in the high field limit were compared with values extracted by fitting of the "low" field data (Fig. 3(a)) and a very good agreement among the two was found.

(ii) Extrapolations of the high field Hall slopes cross zero at zero field at all measured temperatures (see the dotted eye-guide line in Fig. 3(b)) within experimental accuracy. This is fully consistent with the two layer model but opposes an alternative magnetic interpretation of non-linear Hall resistance based on the extraordinary Hall effect. ${ }^{11}$

Mobility and sheet density of holes in Ge substrate layer, calculated from the extracted fitting parameters for samples $\mathrm{GeMn}_{2}$ and $\mathrm{GeMn}_{4} \mathrm{~A}$, are shown in Fig. 4 as a function of temperature. The figure also presents the mobility and holes density extracted from measurements of $\mathrm{Ge}$ wafer
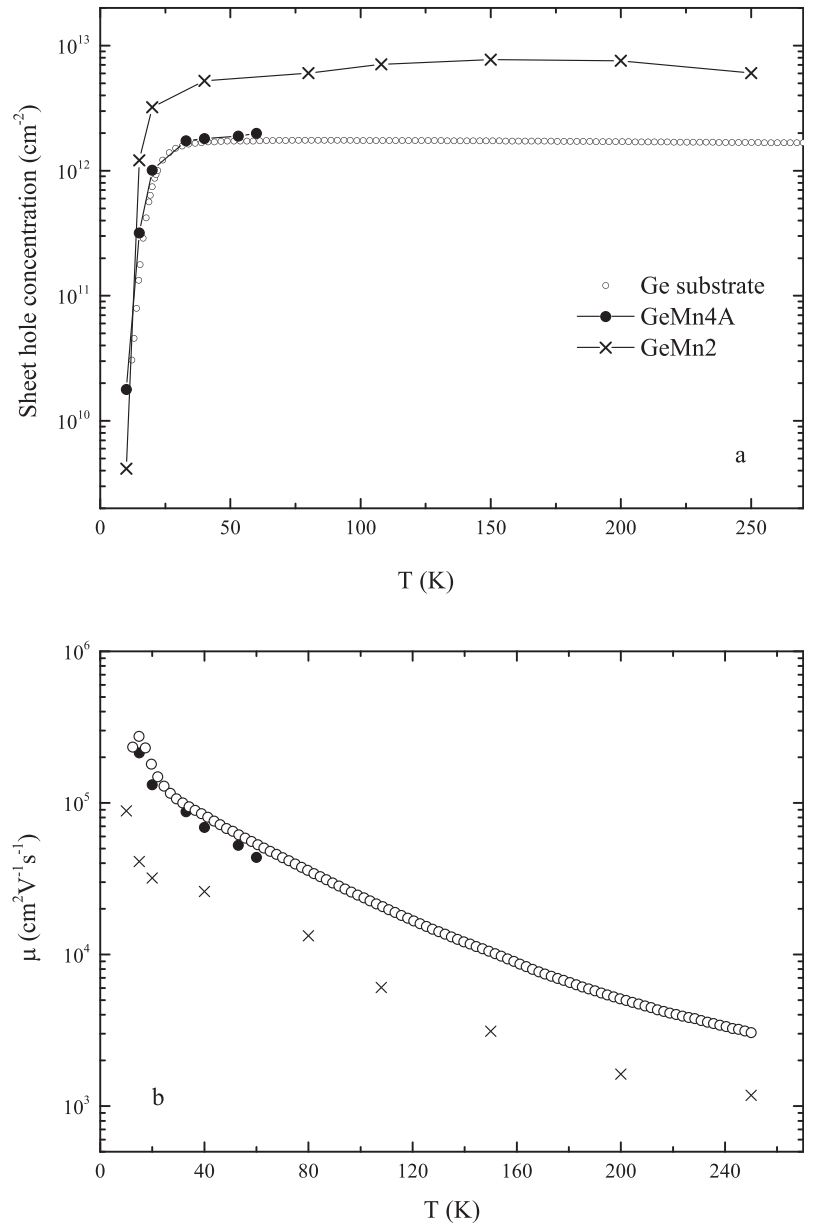

FIG. 4. Temperature dependence of sheet density (a) and mobility (b) of holes in Ge substrate layer in $\mathrm{GeMn}_{2}, \mathrm{GeMn}_{4} \mathrm{~A}$, and $\mathrm{Ge}$ wafer samples. The values for $\mathrm{GeMn}_{2}$ and $\mathrm{GeMn}_{4} \mathrm{~A}$ were calculated by fitting the data by Eq. (4). sample. For this reference sample, the Hall resistance is close to be linear in field at all temperatures, and a single charge carrier density has been calculated directly from the Hall factor. As one sees in Fig. 4, an agreement between the parameters extracted from the two layers model and those measured in Ge wafer sample is very good. Sheet holes concentration (of about $5 \cdot 10^{12} \mathrm{~cm}^{-2}$ in $\mathrm{GeMn}_{2}$, and $10^{12} \mathrm{~cm}^{-2}$ in $\mathrm{GeMn}_{4} \mathrm{~A}$ and Ge samples) is approximately constant down to $25 \mathrm{~K}$ and drops sharply at lower temperatures. Mobility increases with decreasing temperature from about $10^{3} \mathrm{~cm}^{2} / \mathrm{Vs}$ at $250 \mathrm{~K}$ to about $10^{5} \mathrm{~cm}^{2} / \mathrm{Vs}$ at $25 \mathrm{~K}$ in $\mathrm{GeMn}_{2}$ sample and between $2 \cdot 10^{3}$ to $2 \cdot 10^{5} \mathrm{~cm}^{2} / \mathrm{Vs}$ in $\mathrm{GeMn}_{4} \mathrm{~A}$ and Ge samples. The difference in absolute values among the samples can be explained due to different Ge wafers used during the fabrication or due to variations in the effective thickness of the implanted layers. Nevertheless, the deviations are relatively small and the temperature dependence for all samples is similar in a wide temperature range. For the doped GeMn layer, we obtained the sheet holes concentrations of about $5 \cdot 10^{13}$ and $5 \cdot 10^{12} \mathrm{~cm}^{-2}$ and mobility of about $200 \mathrm{~cm}^{2} \mathrm{~V}^{-1} \mathrm{~s}^{-1}$ and $400 \mathrm{~cm}^{2} \mathrm{~V}^{-1} \mathrm{~s}^{-1}$ for $\mathrm{GeMn}_{2}$ and $\mathrm{GeMn}_{4} \mathrm{~A}$ samples, respectively (not shown in figure). Both concentration and mobility of carrier in the doped layer do not change significantly with temperature.

Parameters extracted from the Hall data fitting were used to calculate individual resistances of Ge substrate and the doped MnGe layer of the sandwich samples. The calculated resistances are shown in Fig. 5 as a function of temperature, together with the measured total resistance of $\mathrm{GeMn}_{2}$ sample. Clearly, the calculated resistance of Ge layer in the implanted sample is in a very good agreement with the measured Ge wafer sample data (see Fig. 1). Not less important evidence for validity of the model is that the calculated resistance of the doped $\mathrm{MnGe}$ layer matches the value measured in $\mathrm{GeMn}_{2}$ sample at $4.2 \mathrm{~K}$, where the $\mathrm{MnGe}$ layer remains the only conducting component. Resistance of $\mathrm{Ge}$ substrate layer is metallic-like with a positive resistancetemperature coefficient $d R / d T>0$ down to freezing at $25 \mathrm{~K}$, it is lower than that of $\mathrm{MnGe}$ layer in a wide temperature range between about $15 \mathrm{~K}$ and $220 \mathrm{~K}$ (the difference is

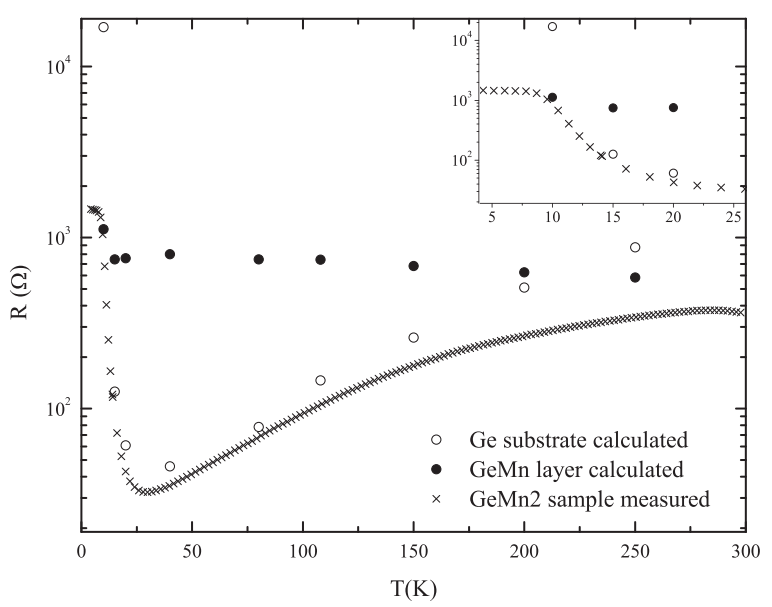

FIG. 5. Temperature dependence of the measured resistance of $\mathrm{GeMn}_{2}$ sample and calculated individual resistances of Ge substrate and the doped MnGe layer. Inset is a zoom of the low temperature range. 
almost two orders of magnitude at $30 \mathrm{~K}$ ). In this temperature range, resistance of the entire sample is determined by $\mathrm{Ge}$ substrate. Resistance of the doped MnGe layer shows a slight insulator-like temperature dependence $(d R / d T<0)$ at all temperatures. Above $220 \mathrm{~K}$ (and below $15 \mathrm{~K}$ ) resistance of the doped layer is lower than that of Ge substrate and thus determines the total resistance of the implanted sample. Similar results were also found in $\mathrm{GeMn}_{4} \mathrm{~A}$ sample, which has higher resistance of the doped layer and, respectively, higher cross-over temperature. Transition in current carrying dominance between the layers with a positive $d R / d T$ (Ge substrate) to a negative one (doped GeMn layer) can be the origin of frequently observed humps in resistance of GeMn samples that were previously assumed to indicate the ferromagnetic ordering in Mn-rich GeMn clusters. ${ }^{11}$

To summarize this part: the model presenting two parallel conducting layers where the first layer is a thick Ge substrate with a relatively low density of highly mobile carriers and the second layer is a relatively thin Mn doped Ge with higher density of low mobility carriers provides a good description of a zero field temperature dependent resistance and a non-monotonous Hall effect, as observed in $\mathrm{Mn}$ implanted Ge samples.

\section{MAGNETORESISTANCE}

\section{A. Two-carriers model}

There are two ways to analyze the magnetoresistance in the framework of the two layers model. The first is by fitting the magnetoresistance data by Eq. (3) using the measured $\rho_{0}$ and fitting parameters $\rho_{\infty}$ and $B_{s}$. Another, and probably more illustrious procedure, is to calculate the magnetoresistance predicted by the model by using parameters extracted from the Hall data fitting. We used the measured values of $\rho_{0}$ and $R_{0}$ and parameters $R_{\infty}$ and $B_{s}$ derived from Hall data fitting to calculate parameters $\rho_{1}, \rho_{2}, R_{1}, R_{2}$, and $\rho_{\infty}$ and then calculated the magnetoresistance by using Eq. (3). Fig. 6 presents the magnetoresistance of $\mathrm{GeMn}_{2}$ sample measured up to $60 \mathrm{~T}$ that allows us to analyze the model in a very wide field range. The dotted (blue) line is the best fit to the magnetoresistance data calculated using Eq. (3), and the solid line (red) is the predicted magnetoresistance calculated using the parameters extracted from the Hall data fitting (Eq. (4)). Fig. 6 (b) is a zoom of a low field range. The shown data and calculations are for $25 \mathrm{~K}$ but the results are typical for all temperatures. Mobility and density of carriers extracted from fitting of magnetoresistance differ significantly from those calculated from the Hall data. More important is that contrary to both calculations the measured data shows no signs of the expected saturation in the high fields limit and increases sharply at low fields without following the predicted quadratic field dependence. The range of quadratic dependence, if existing, is below $10^{-2} \mathrm{~T}$, the field resolution in our experiments.

We made an effort to resolve the low field discrepancy by assuming the presence of a third low mass and high mobility carrier in Ge substrate. Light carriers can be affected by a relatively low field thus reducing the range of the quadratic field dependence. Presence of light and heavy carriers with mass ratio $\frac{m_{\text {light }}^{*}}{m_{\text {m }}^{*}}=0.1298$ has been demonstrated in two-band Ge. ${ }^{18}$ The Theavy $^{2}$ results of the three-carrier fitting (not shown here) are qualitatively better than those of the two carriers model, which is not surprising due to a large number of fitting parameters. However, discrepancies among the measured and calculated data in the low and high field ranges remain unresolved.

\section{B. Angle-dependent magnetoresistance}

Lorentz force acting on charge carriers depends on an angle between the charge velocity and magnetic field; therefore, the magnetotransport effects related to Lorentz force should depend strongly on field tilting and disappear in an ideal case of field strictly parallel to the current line. Fig. 7 presents the magnetoresistance of $\mathrm{GeMn}_{2}$ sample measured at several temperatures with field applied normal to the sample plane (a), and approximately in-plane parallel to current
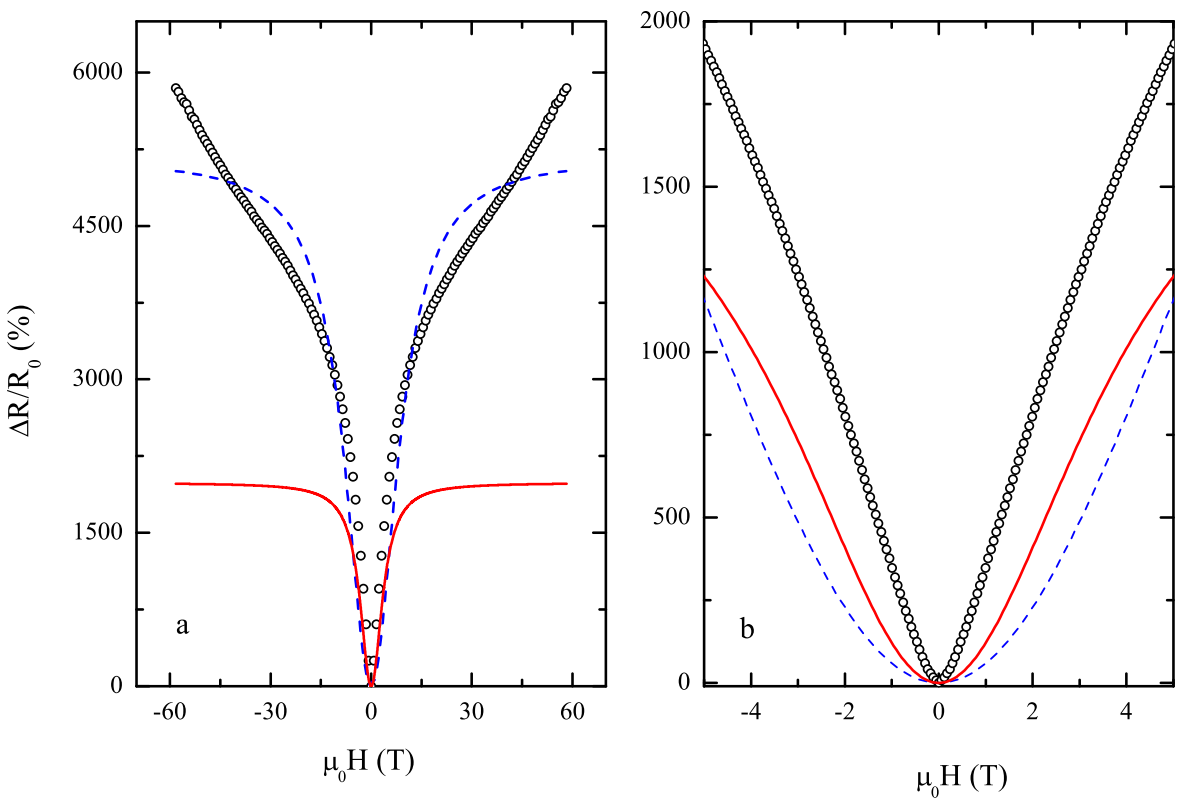

FIG. 6. Magnetoresistance of $\mathrm{GeMn}_{2}$ sample as a function of applied field. The dotted (blue) line is the best fit to the magnetoresistance data calculated using Eq. (3). The solid line (red) is the predicted magnetoresistance calculated using parameters extracted from the Hall data fitting (Eq. (4)). (b) is a zoom of a low field range. $\mathrm{T}=25 \mathrm{~K}$. 

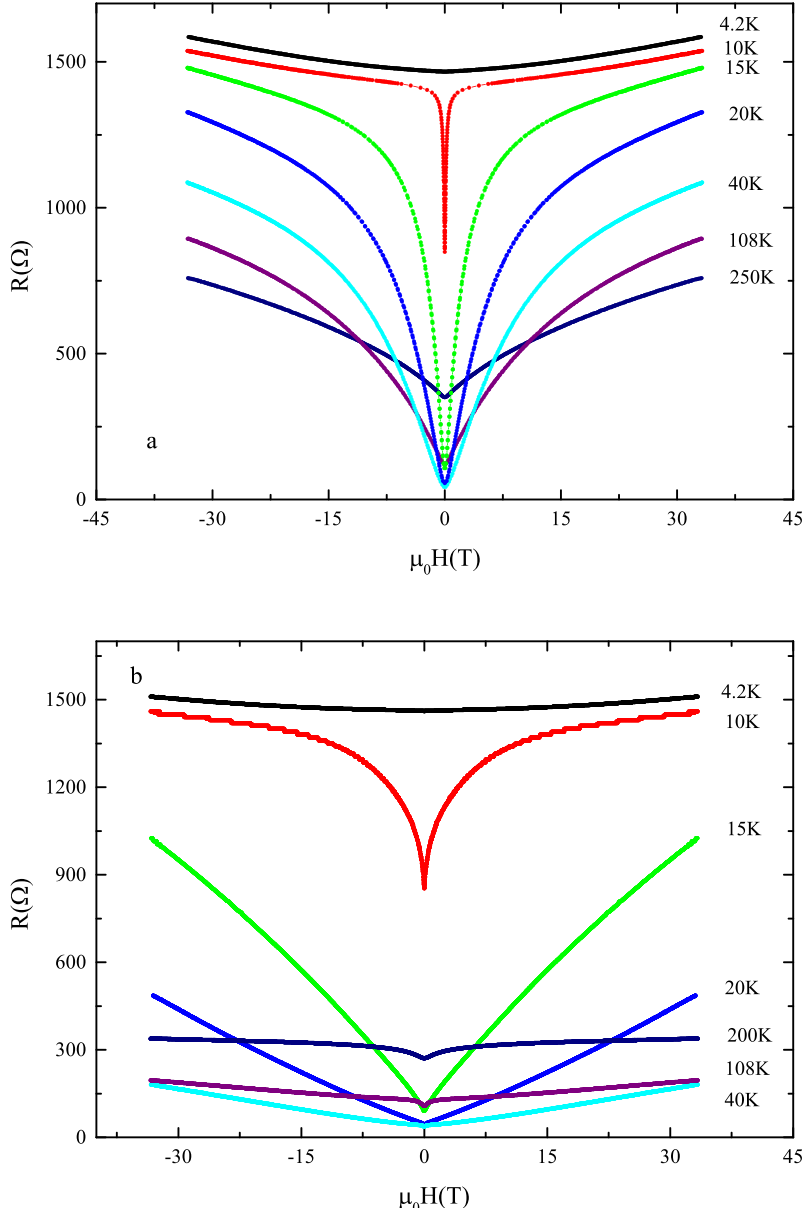

FIG. 7. Magnetoresistance of $\mathrm{GeMn}_{2}$ sample measured at several temperatures with field applied normal to the sample plane (a), and approximately in-plane parallel to current leads orientation (b).

leads orientation (b) (we shall call this orientation quasi-parallel). Significant magnetoresistance is observed in the second case as well. The setup alignment of the sample relative to field orientation is not precise. Since all quasi-parallel measurements were performed in the same setup, one can estimate the deviation of the field from the in-plane orientation by comparing the Hall voltage slopes measured at $4.2 \mathrm{~K}$ in the perpendicular and the quasi-parallel orientations. As noted above, at $4.2 \mathrm{~K}$, the doped GeMn layer is the only conducting one and its Hall signal is linear and proportional to the field component normal to the plane. Thus, we found that the tilting-from-plane angle in the quasi-parallel series of measurements is $2.2^{\circ}$.

Fig. 8 presents the attempts to rescale magnetoresistance measured in the transverse $\left(R_{\perp}\right)$ and quasi-parallel $\left(R_{\| *}\right)$ field orientations as a function of field projection perpendicular to the sample plane for three selected temperatures: $200 \mathrm{~K}, 80 \mathrm{~K}$, and $10 \mathrm{~K}$. An effective tilting angle $\theta^{*}$ and the respective normal-to-plane field projection in the quasi-parallel measurements are defined via resistance values reached in the quasi-longitudinal experiments at $30 \mathrm{~T}$ field as: $R_{\| *}(30 \mathrm{~T})$ $=R_{\perp}\left(B \sin \theta^{*}\right)$. The two orientations data sets scale quite well at $80 \mathrm{~K}$ (excluding the low field range) but differ strongly at high $(200 \mathrm{~K})$ and low $(10 \mathrm{~K})$ temperatures. At high temperatures, the quasi-longitudinal magnetoresistance grows quicker than expected from the field scaling, while the opposite
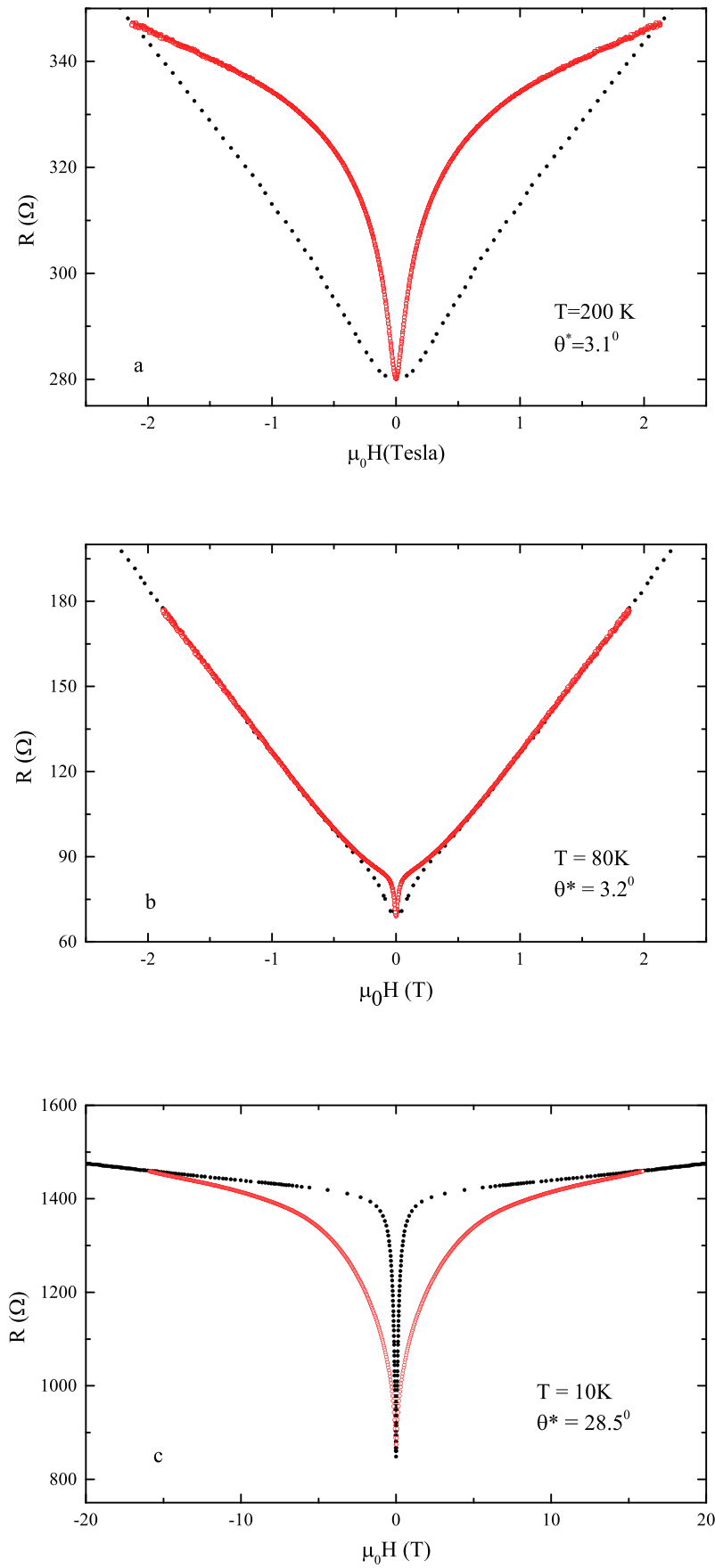

FIG. 8. Magnetoresistance measured in the transverse field orientation ( $R_{\perp}$ - solid black circles) and rescaled magnetoresistance obtained for quasi-parallel ( $R_{\| *}$-open circles) field orientations as a function of field projection perpendicular to the sample plane for: $200 \mathrm{~K}$ (a), $80 \mathrm{~K}$ (b), and $10 \mathrm{~K}(\mathrm{c})$. An effective tilting angle $\theta^{*}$ and the respective normal-to-plane field projection in the quasi-parallel measurements are defined via resistance values reached in the quasi-longitudinal experiments at $30 \mathrm{~T}$ as: $R_{\| *}(30 T)=R_{\perp}\left(B \sin \theta^{*}\right)$.

appears at low temperatures. An effective tilting angle $\theta^{*}$ calculated for different temperatures is shown in Fig. 9. In the intermediate temperature range, $\theta^{*}$ is close to the deviation angle extracted from the Hall slope measurements $\left(2.2^{\circ}\right)$. At low and high temperatures, $\theta^{*}$ changes with temperature and its deviation from $2.2^{\circ}$ grows significantly.

One can thus conclude that the quasi-longitudinal measurements are reasonably consistent with the two-layers 


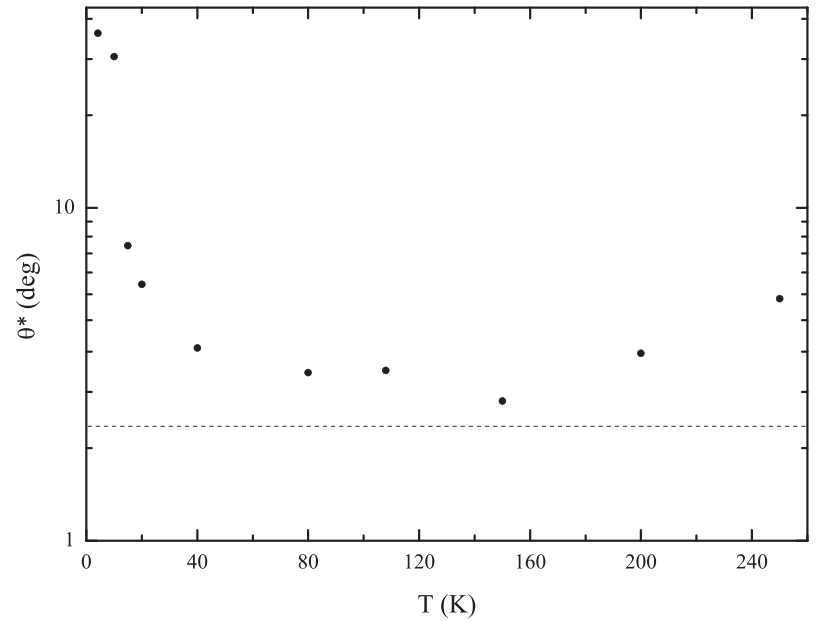

FIG. 9. Effective tilting angles $\theta^{*}$ calculated for different temperatures. The dotted horizontal line indicates the tilting angle of $2.2^{\circ}$ extracted from the Hall slope measurements. Vertical axis is in logarithmic scale.

model at intermediate temperatures $(50 \mathrm{~K}<\mathrm{T}<150 \mathrm{~K})$ and are inconsistent at low and high temperatures. Non-orbital mechanisms giving rise to longitudinal magnetoresistance in parallel to current field are dominant at low and high temperatures.

\section{Magnetoresistance of Ge}

So far we limited our discussion to a simple case of two (three) carriers in two conducting layers while neglecting intrinsic magnetoresistance of each layer. However, intrinsic magnetoresistance of $\mathrm{Ge}$ substrate is essential, as can be appreciated from Fig. 10. Here, we show the low field magnetoresistance of Ge wafer sample and that of the $\mathrm{GeMn}_{4} \mathrm{~A}$ sample at room and $77 \mathrm{~K}$ temperatures. Both samples demonstrate identical behavior at low fields at both temperatures, therefore the low field magnetoresistance of implanted MnGe samples should be associated with that of Ge substrate. At room temperature, magnetoresistance looks quadratic in field up to about $0.3 \mathrm{~T}$ in both samples. At $77 \mathrm{~K}$, resistance increases sharply and linearly with field and no low field quadratic range can be identified within an accuracy of the experiment.

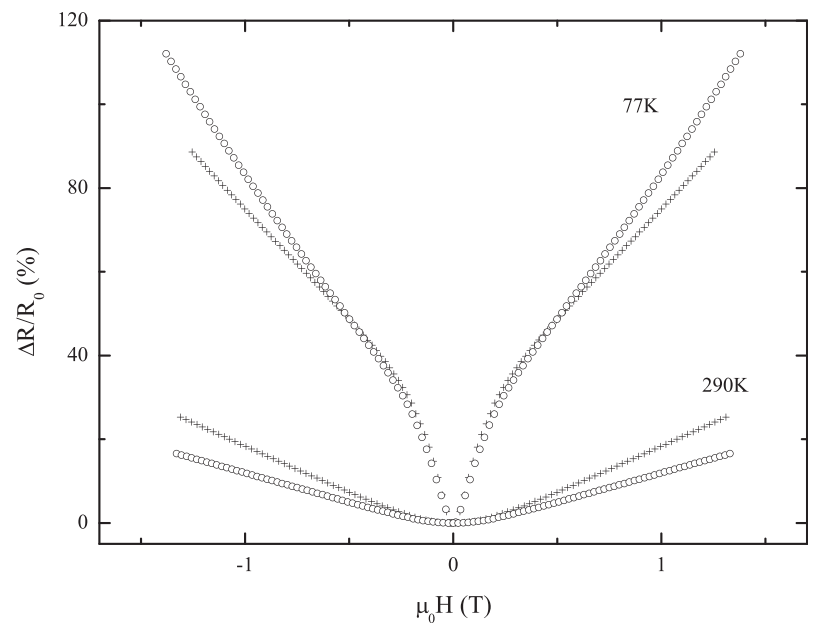

FIG. 10. Low field magnetoresistance of Ge substrate sample (O) and of $\mathrm{GeMn}_{4} \mathrm{~A}$ sample $(\times)$ at room and $77 \mathrm{~K}$ temperatures.

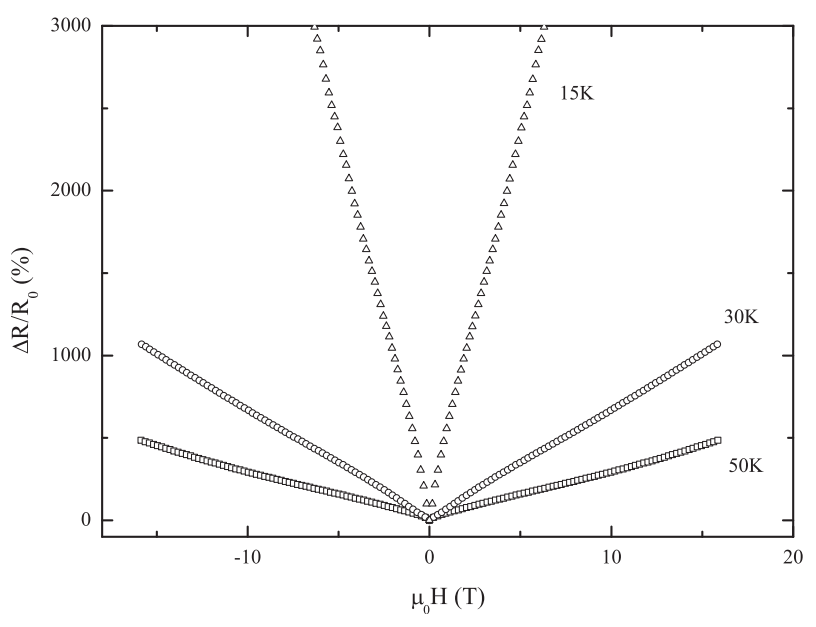

FIG. 11. Magnetoresistance of Ge wafer sample in fields up to $16 \mathrm{~T}$ at $15 \mathrm{~K}$, $30 \mathrm{~K}$, and $50 \mathrm{~K}$. Field is applied normal to the sample plane.

Fig. 11 presents the magnetoresistance of Ge wafer sample in fields up to $16 \mathrm{~T}$. Notably, it is linear in field and grows dramatically in the vicinity of Ge freezing temperature. Magnetoresistance of p-type germanium remains an open question for more than half a century. Its source has been searched in the framework of two valence bands having carriers of effective mass approximately $0.3 \mathrm{~m}_{\mathrm{o}}$ and $0.04 \mathrm{~m}_{\mathrm{o}}$, respectively. ${ }^{18,19}$ However, two principal discrepancies with the model have been identified from the very beginning: ${ }^{20}$ the low-field Hall effect data gave values for the mobilities of the two kinds of carriers, which were incompatible with the observed field dependence of the magnetoconductivity, and the resistivity did not saturate at high fields as would be expected according to classical transport theory. Instead, the magnetoresistance of $\mathrm{Ge}$ was found to be linear in field and non-saturating up to the highest fields available. ${ }^{21}$ Similar behavior was also found in n-type $\mathrm{Ge},{ }^{22}$ while the ratio of the magnetoresistance in p-type and n-type Ge being independent of temperature. It was suggested that the measured puzzling magnetoresistance does not reflect any difference in the structures of the conduction and valence bands of Ge. Similar linear behavior has also been observed in other high mobility semiconductors, such as $\mathrm{InSb}^{23,24}$ and PbTe. ${ }^{25,26} \mathrm{~A}$ renewed interest in strong linear magnetoresistance has been triggered by observation of the phenomenon in doped silver chalcogenides, ${ }^{27,28}$ followed by similar observations in $\mathrm{InSb}^{29}$ and InAs. ${ }^{30}$ Current interpretation of the effect is associated with strong disorder, where current paths deviate from the applied voltage due to spatial fluctuations of Hall mobility. ${ }^{31,32}$ Simulations of large spatial conductivity fluctuations in strongly inhomogeneous semiconductors derived a linear magnetoresistance picked up from the Hall voltage along macroscopically distorted current paths. The phenomenon appears to be general for variety of materials, although the model leaves a number of open questions.

(1) Linear magnetoresistance is expected to take place at high fields when $\mu B \gg 1$. Mobility of Ge found in our sample at $77 \mathrm{~K}$ is about $1 \frac{\mathrm{m}^{2}}{\mathrm{Vcm}}$; therefore, an onset of linearity can be expected at fields above $1 \mathrm{~T}$. In practice, as shown in Fig. 11. the quasi-linear magnetoresistance 


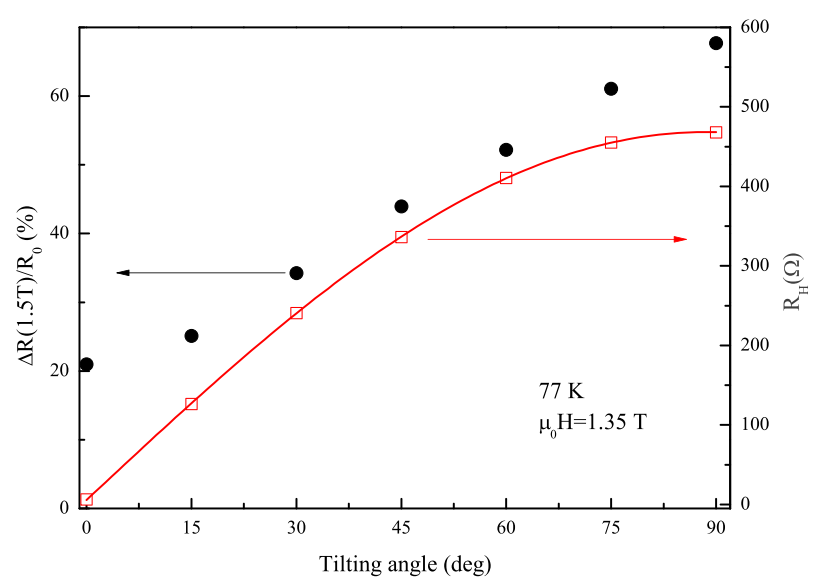

FIG. 12. Magnetoresistance and Hall resistance measured at 1.5 T in Ge substrate sample at $77 \mathrm{~K}$ as a function of the off-plane angle $\theta$ between the current leads orientation and the applied field direction. $\theta=0$ corresponds to the in-plane field orientation and $90^{\circ}$ correspond to the transverse one.

starts already below $10^{-2} \mathrm{~T}$ (within an experimental accuracy of low field resolution). Similar low field onset of linearity has been reported in silver chalcogenides ${ }^{33}$ and can be traced in data on other materials. ${ }^{30}$

(2) The disorder models predict strong linear magnetoresistance in transverse field orientation but small and even negative longitudinal magnetoresistance when field is applied parallel to current. ${ }^{33,34}$ This prediction is not met in Ge. Fig. 12 presents the magnetoresistance and Hall resistance measured at $1.5 \mathrm{~T}$ in the Ge wafer sample at $77 \mathrm{~K}$ as a function of the off-plane angle $\theta$ between the current leads orientation and the applied field direction. Zero angle corresponds to the field-in-plane orientation and $90^{\circ}$ correspond to the transverse one. The Hall voltage amplitude scales, as expected, with field projection normal to plane and follows the $\sin \theta$ dependence (solid line in Fig. 13). The magnetoresistance does not follow the trend. The longitudinal MR is significant, being about a third of the transverse one. Similar ratio between longitudinal and transverse effects has been previously found in p-type Ge. ${ }^{20}$

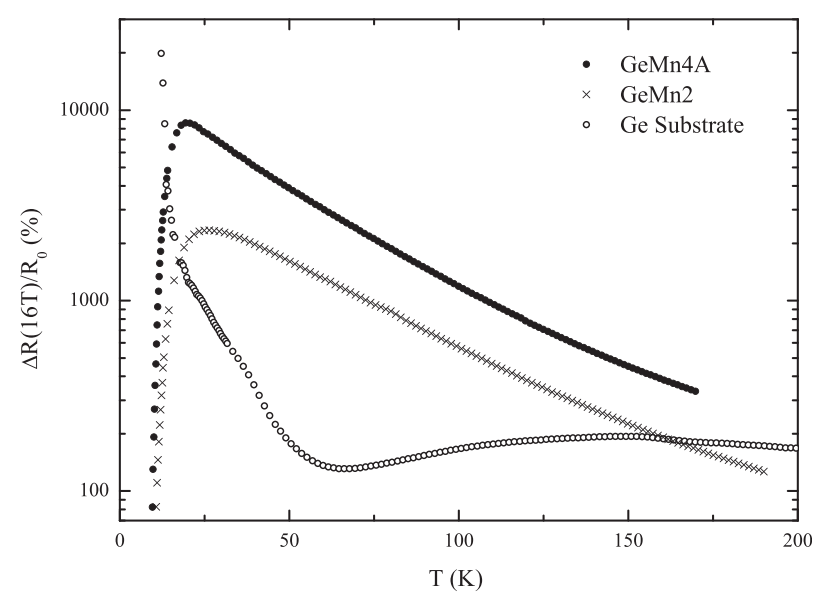

FIG. 13. Magnetoresistance of $\mathrm{GeMn}_{2}, \mathrm{GeMn}_{4} \mathrm{~A}$, and Ge wafer samples as a function of temperature. MR is defined as $\frac{\Delta R(16 T)}{R}=\frac{R(16 T)-R(0)}{R(0)}$.
(3) Magnitude of the transverse magnetoresistance and its temperature dependence we find in our samples is very close to values observed by, e.g., Gallagher in his single crystals in $1968 .^{21}$ It is hard to imagine that random disorder in single crystals fabricated half a decade ago is identical to that in commercial Ge wafers we used, to give rise to the same magnetoresistance values.

Whatever the physical mechanism is behind the intrinsic magnetoresistance of $\mathrm{Ge}$, it is interesting to identify its contribution to the total magnetoresistance of the implanted samples. At room temperature, magnetoresistance of $\mathrm{Ge}$ increases sharper than that of the implanted sample and reaches $30 \%$ at $1.5 \mathrm{~T}$ field (Fig. 10). At $77 \mathrm{~K}$, the magnetoresistance of the implanted sample is $115 \%$ and exceeds that of Ge $(90 \%)$. The high field magnetoresistance of both samples is shown in Fig. 13 as a function of temperature, where MR is defined as $\frac{\Delta R}{R}=\frac{R(16 T)-R(0)}{R(0)}$. MR of Ge remains approximately constant $(100 \%-200 \%)$ in a wide temperature range down to $60 \mathrm{~K}$ and increases sharply below $50 \mathrm{~K}$. Notably, increase of magnetoresistance starts at temperature significantly higher than the freezing temperature of zero field resistance $(25 \mathrm{~K}$ see Fig. 1). MR of Ge exceeds that of the implanted samples at low temperatures, and also at room temperature where resistance of Ge substrate is higher than that of the doped GeMn layer (see Fig. 5). A competition between an intrinsic Ge MR and the two layers MR mechanism can be understood since the latter is strong when the high mobility layer has resistance smaller than the low mobility one. The high mobility charge gets localized by magnetic field giving rise to magnetoresistance. When resistance of the high mobility layer grows higher than that of the low mobility one, the latter shunts most of current and resistance of the system saturates. In our case, mobility of Ge substrate is the highest at all temperatures. Therefore, the two-carrier mechanism is dominant at intermediate temperatures where resistance of Ge substrate is lower than that of the doped one. The magnitude of the two-carriers magnetoresistance is determined by a difference between resistances of high mobility Ge and the low mobility GeMn layer. Since resistance of the implanted layer in $\mathrm{GeMn}_{4} \mathrm{~A}$ sample is higher than in $\mathrm{GeMn}_{2}$ one, this leads to respectively higher magnetoresistance. In the room temperature range, the doped GeMn layer has resistance lower than that of Ge substrate and shunts the current. The intrinsic Ge MR and the two layers mechanisms become comparable while the contribution of the latter diminishes at higher temperatures with growing resistance of Ge substrate. The picture is consistent with quasi-longitudinal measurements discussed above. At intermediate temperatures, the quasi-longitudinal data scales reasonably well with the transverse ones indicating the dominance of the two-carriers Lorentz force mechanism. At high and low temperatures, the Lorentz scaling fails due to the dominance of the intrinsic Ge MR.

\section{Magnetoresistance of the implanted GeMn layer}

So, what is the magnetoresistance of the implanted GeMn layer? Explicitly this can be revealed below the freezing temperature of Ge substrate when the doped GeMn layer remains the sole current carrying channel. Fig. 14 presents 


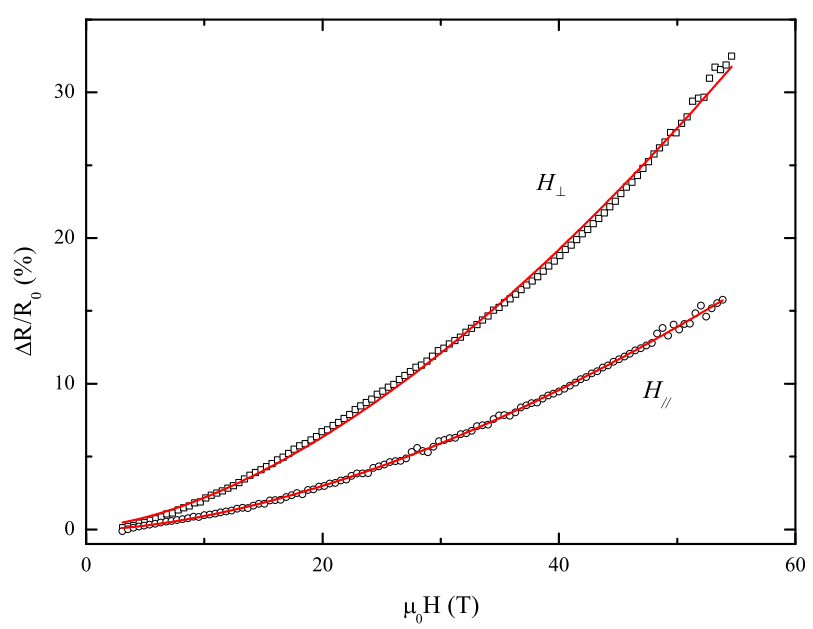

FIG. 14. Magnetoresistance of $\mathrm{GeMn}_{2}$ sample in the transverse ( $\square$ ) and longitudinal $(\mathrm{O})$ field orientations measured at $4.2 \mathrm{~K}$. The only conducting channel is the doped GeMn layer. Solid lines are fits of the form $\Delta R=a H^{n}$ with $\mathrm{n}=1.6 \pm 0.1$ and different coefficients $a$ for two orientations.

the magnetoresistance of $\mathrm{GeMn}_{2}$ sample in the transverse and longitudinal field orientations measured at $4.2 \mathrm{~K}$ in fields up to $55 \mathrm{~T}$. In both orientations, MR follows the form $\frac{\Delta R}{R}$ $=a H^{n}$ with $\mathrm{n} \approx 3 / 2$ in the entire field range with no sign of saturation up to $60 \mathrm{~T}$. The power index varies slightly in different samples in the limits $1.5 \leq \mathrm{n} \leq 2$. The same non-saturating power law behavior can be identified also at higher temperatures in the high field limit (see Fig. 15) when resistance of Ge substrate exceeds resistance of the doped MnGe layer and the latter becomes the major current carrier. The magnitude of the effect is significantly smaller than that generated by intrinsic Ge and the two-layers mechanisms but is far from being negligible. Notably, the fit to a single " $3 / 2$ " power index over a very wide field range is puzzling. Attempts to fit the data by a combination of linear and quadratic field terms were not successful.

It seems that GeMn implanted layer is not the only material that demonstrates the " $3 / 2$ " power MR. Similar behavior has been observed in quasi-one-dimensional organic conductor $(\mathrm{TMTSF})_{2} \mathrm{PF}_{6},{ }^{35}$ in SiMn thin films grown by molecular

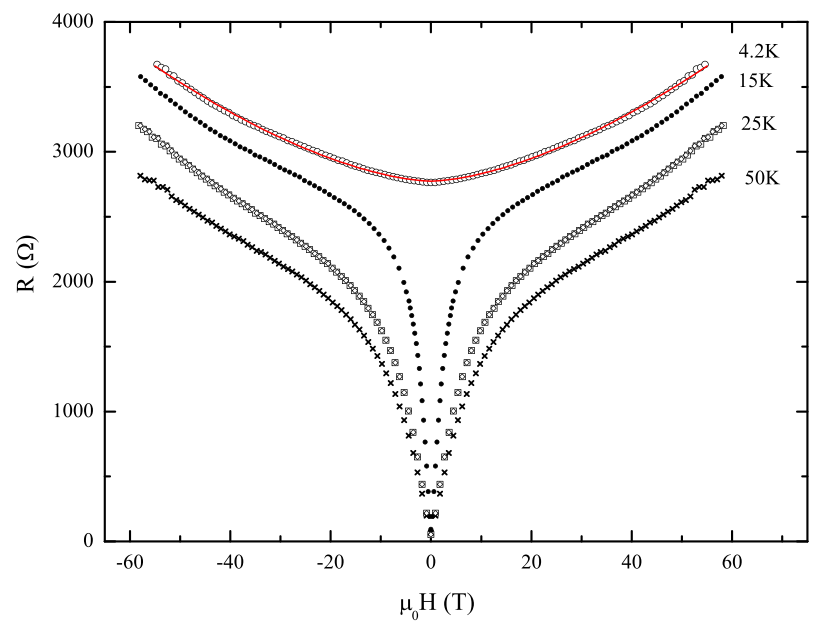

FIG. 15. Magnetoresistance of $\mathrm{GeMn}_{2}$ sample at $4.2,15 \mathrm{~K}$ and $25 \mathrm{~K}$ in fields up to $55 \mathrm{~T}$. Solid line is a fit of the $4.2 \mathrm{~K}$ data to $R(H)=R_{0}+a H^{1.6}$. beam epitaxy, ${ }^{36}$ in $\mathrm{CoC}$ composites, ${ }^{37}$ and permalloy-graphite NiFeC composites. $^{38}$ The only theoretical prediction of the $3 / 2$ power law magnetoresistance, we are aware of, has been drawn for two-dimensional electron gas in the presence of small modulated magnetic field with zero average, ${ }^{39}$ the model that can hardly be relevant to our case.

\section{SUMMARY}

Our main findings can be briefly summarized as following: the zero field resistance and the non-monotonic field dependent Hall effect in Mn implanted Ge can be consistently explained by parallel conductance along thick ptype Ge substrate with low concentration of highly mobile carriers and along thin Mn doped Ge layer with low mobility carriers. The same model is not sufficient to reproduce the observed suppression of quadratic field dependent magnetoresistance at low fields and absence of saturation at high fields. Two additional mechanisms have been identified: a strong quasi-linear magnetoresistance of $\mathrm{Ge}$ substrate that dominates the low field range and a nonsaturating "3/2" power law magnetoresistance of the $\mathrm{Mn}$ doped Ge layer dominating the high field range. Comprehensive understanding of both phenomena is lacking. Magnetoresistance due to parallel conductance along two layers is mostly important when resistance of the high mobility layer is lower than that of the low mobility one, while the magnitude of the effect depends on the ratio between resistances of the two layers. New type of sensitive magnetic field sensors using tailored two-layer conductive structures can thus be anticipated.

\section{ACKNOWLEDGMENTS}

This work was supported by the Air Force Office of Scientific Research, Air Force Material Command, USAF Grant No. FA8655-11-1-3081 and by Russia-Israel Scientific Research Cooperation Grant No. 3-8363.

${ }^{1}$ H. H. Woodbury and W. W. Tyler, Phys. Rev. 100, 659 (1955).

${ }^{2}$ S. Cho, S. Choi, S. C. Hong, Y. Kim, J. B. Ketterson, B. J. Kim, Y. C. Kim, and J. H. Hung, Phys. Rev. B 66, 033303 (2002).

${ }^{3}$ Y. D. Park, A. Wilson, A. T. Hanbicki, J. E. Mattson, T. Ambrose, G. Spanos, and B. T. Jonker, Appl. Phys. Lett. 78, 2739 (2001).

${ }^{4}$ C. Bihler, C. Jaeger, T. Vallaitis, M. Gjukic, M. S. Brandt, E. Pippel, J. Woltersdorf, and U. Goesele, Appl. Phys. Lett. 88, 112506 (2006).

${ }^{5}$ S. Zhou, A. Shalimov, K. Potzger, N. M. Jeutter, C. Baehtz, M. Helm, J. Fassbender, and H. Schmidt, Appl. Phys. Lett. 95, 192505 (2009).

${ }^{6}$ Y. Wang, J. Zou, Z. Zhao, X. Han, X. Zhou, and K. L. Wang, Appl. Phys. Lett. 92, 101913 (2008).

${ }^{7}$ M. Jamet, A. Barski, T. Devillers, V. Poydenot, R. Dujarin, P. BayleGuillemaud, J. Rothman, E. Bellet-Amalric, A. Marty, J. Cibert, R. Mattana, and S. Tatarenko, Nature Mater. 5, 653 (2006).

${ }^{8}$ I.-S. Yu, M. Jamet, A. Marty, T. Devillers, A. Barski, C. Beigne, P. BayleGuillemaud, and J. Cibert, J. Appl. Phys. 109, 123906 (2011).

${ }^{9}$ A. P. Li, C. Zeng, K. van Benthem, M. F. Chisholm, J. Shen, S. V. S. Nageswara Rao, S. K. Dixit, L. C. Feldman, A. G. Petukhov, M. Foygel, and H. H. Weitering, Phys. Rev. B 75, 201201 (2007).

${ }^{10}$ M. Foygel and A. G. Petukhov, Phys. Rev. B 76, 205202 (2007).

${ }^{11}$ O. Riss, A. Gerber, I. Y. Korenblit, A. Suslov, M. Passacantando, and L. Ottaviano, Phys. Rev. B 79, 241202(R) (2009).

${ }^{12}$ E. Z. Meilikhov and R. M. Farzetdinova, J. Appl. Phys. 109, 053906 (2011). 
${ }^{13}$ S. Zhou, D. Burger, M. Helm, and H. Schmidt, Appl. Phys. Lett. 95, 172103 (2009).

${ }^{14}$ N. Sircar, S. Ahlers, C. Majer, G. Abstreiter, and D. Bougeard, Phys. Rev. B 83, 125306 (2011).

${ }^{15}$ L. Ottaviano, M. Passacantando, A. Verna, P. Parisse, S. Picozzi, G. Impellizzeri, and F. Priolo, Phys. Status Solidi A 204, 136 (2007).

${ }^{16}$ M. Passacantando, L. Ottaviano, F. D’Orazio, F. Lucari, M. De Biase, G. Impellizzeri, and F. Priolo, Phys. Rev. B 73, 195207 (2006).

${ }^{17}$ R. L. Jones and P. Fisher, Phys. Chem. Solids 26, 1125 (1965).

${ }^{18}$ G. Dresselhaus, A. F. Kip, and C. Kittel, Phys. Rev. 95, 568 (1954).

${ }^{19}$ R. N. Dexter, H. J. Zeinger, and B. Lax, Phys. Rev. 104, 637 (1956).

${ }^{20}$ C. Goldberg, E. N. Adams, and R. E. Davis, Phys. Rev. 105, 865 (1957).

${ }^{21}$ J. W. Gallagher, Phys. Rev. 171, 987 (1968).

${ }^{22}$ J. W. Gallagher and W. F. Love, Phys. Rev. 161, 793 (1967).

${ }^{23}$ J. C. Haslett and W. F. Love, J. Phys. Chem. Solids 8, 518 (1959).

${ }^{24}$ W. Schneider, H. Bruhms, and K. Hubner, J. Phys. Chem. Solids 41, 313 (1980).

${ }^{25}$ Y. Kanai, R. Nii, and N. Watanabe, J. Appl. Phys. 32, 2146 (1961).

${ }^{26}$ R. S. Allgaier, J. B. Restorff, and B. Houston, J. Appl. Phys. 53, 3110 (1982).
${ }^{27}$ R. Xu, A. Husmann, T. F. Rosenbaum, M. L. Saboungi, J. E. Enderby, and P. B. Littlewood, Nature (London) 390, 57 (1997).

${ }^{28}$ M. Lee, T. F. Rosenbaum, M. L. Saboungi, and H. S. Schnyders, Phys. Rev. Lett. 88, 066602 (2002).

${ }^{29}$ J. Hu and T. F. Rosenbaum, Nature Mater. 7, 697 (2008).

${ }^{30}$ N. V. Kozlova, N. Mori, O. Makarovsky, L. Eaves, Q. D. Zhuang, A. Krier, and A. Patanè, Nat. Commun. 3, 1097 (2012).

${ }^{31}$ C. Herring, J. Appl. Phys. 31, 1939 (1960).

${ }^{32}$ M. M. Parish and P. B. Littlewood, Nature (London) 426, 162 (2003).

${ }^{33}$ J. Hu, T. F. Rosenbaum, and J. B. Betts, Phys. Rev. Lett. 95, 186603 (2005).

${ }^{34}$ J. Hu, M. M. Parish, and T. F. Rosenbaum, Phys. Rev. B 75, 214203 (2007).

${ }^{35}$ G. Kriza, G. Szegny, I. Kezsmarki, and G. Mihaly, Phys. Rev. B 60, R8434 (1999).

${ }^{36}$ T. T. Lan Anh, H. K. Lim, B. C. Lee, D. H. Kim, K. J. Baek, Y. E. Ihm, D. J. Kim, H. J. Kim, and J. H. Kim, J. Korean Phys. Soc. 55, 309 (2009).

${ }^{37}$ Q. Z. Xue, X. Zhang, and D. D. Zhu, Physica B 334, 216 (2003).

${ }^{38}$ G. Mandala, V. Srinivas, and V. V. Rao, J. Alloys Compd. 484, 851 (2009).

${ }^{39}$ A. Matulis and F. M. Peeters, Phys. Rev. B 62, 91 (2000). 